Revista Perspectivas Online: Exatas \& Engenharias

Dezembro de 2017, Vol.7, n 19 , p. 42-51

ISSN: $2236-885 \mathrm{X}$ (Online)

DOI: $10.25242 / 885 \times 7192017994$

\title{
SIMULAÇÃO DO PROCESSO DE ATENDIMENTO DE UM RESTAURANTE UNIVERSITÁRIO: UM ESTUDO DE CASO
}

\section{RESUMO}

MONTEIRO, C.I; SANTOS, J.A.A.; SCHIMIDT, A.P. Simulação do processo de atendimento de um restaurante universitário: um estudo de caso. Perspectivas Online: Exatas \& Engenharias, v. 7, n.19, p.42$51,2017$.

Existem vários fatores que determinan a satisfação dos clientes em um restaurante. Um desses fatores é o número de clientes nas filas do restaurante. Atendendo a necessidade de diminuir as filas, o trabalho aqui desenvolvido teve como objetivo analisar o processo de atendimento de um restaurante universitário. A metodologia utilizada é a de modelagem através de simulação computacional, de caráter quantitativo e caracterizada como participativa. Um modelo do tipo dinâmico, discreto e estocástico foi implementado no software de simulação Arena ${ }^{\circledR}$. O modelo foi validado e análise de filas foram realizadas. Identificou-se que a fila do caixa tem maior impacto no tempo permanência dos clientes no restaurante. Observou-se também, de resultados obtidos de simulação, uma utilização dos caixas de 100\% durante $\quad 0$ período estudado.

Palavras-chave: Simulação discreta; Arena; Filas.

\begin{abstract}
There are several factors that determine customer satisfaction in a restaurant. One of these factors is the number of customers in the restaurant queues. Given the need to reduce queues, the work developed here had the objective of analyzing the process of attending a university restaurant. The methodology used is that of modeling through computational simulation, whose quantitative character and

characterized as participatory. A dynamic, discrete and stochastic model was implemented in the Arena ${ }^{\circledR}$ simulation software. The model was validated and queuing analysis was performed. It has been identified that the queue of the cashier has greater impact on the time guests stay in the restaurant. It was also observed, from results obtained from simulation, a use of the boxes of $100 \%$ during the studied period.

Keywords: Discrete simulation; Arena; Queues.

\footnotetext{
${ }^{1}$ Universidade Tecnológica Federal do Paraná - Discente do Curso de Engenharia de Produção - Av. Sete de Setembro, 3165, Rebouças, Curitiba, PR, CEP: 80230-901, Brasil;

${ }^{2}$ Universidade Tecnológica Federal do Paraná - Coordenacao do Curso de Engenharia de Produção - Av. Sete de Setembro, 3165, Rebouças, Curitiba, PR, CEP: 80230-901, Brasil;

(*)e-mail: ciroim93@gmail.com

Data de chegada: 02/07/2016 Aceito para publicação: 03/12/2017
}

Persp. Online: exatas \& eng., Campos dos Goytacazes, 19 (07) 42-51 - 2017 


\section{INTRODUÇÃO}

O setor de serviços está em constante crescimento, dentre alguns fatores deste crescimento pode-se citar a busca do homem por conforto e comodidade. Atualmente a área de serviços é tida como a área que apresenta maior crescimento no setor econômico mundial. O setor de alimentação tem grande destaque no mercado brasileiro, tendo em vista que a alimentação é essencial para o desenvolvimento de todo ser humano (ALVES, 2012).

Com o aumento das jornadas de trabalho, as pessoas já não tem mais tempo para preparar seu próprio alimento, fazendo com que busquem um local para realizar suas refeições. Os restaurantes self-service surgem como empreendimentos alimentícios que oferecem comida pronta, variada, nutritiva, a um preço acessível e na quantidade desejada pelo consumidor (ALVES, 2012).

Nos centros universitários, restaurantes desta categoria são predominantes, todavia, devido à grande demanda, que pode incluir: alunos, professores, colaboradores e até moradores da região, a geração de filas torna-se comum, e um desgaste para os que necessitam deste serviço.

Banks (2009), salienta que a formação de filas é dada pelo aumento dos consumidores, e pela incapacidade do sistema em atender a demanda. As técnicas de simulação surgem como ferramentas que buscam encontrar um ponto de equilíbrio entre a satisfação dos clientes, e a viabilidade econômica para o provedor do serviço.

A simulação computacional é uma ferramenta que possibilita um fácil entendimento da dinâmica operacional de um sistema, sem realizar alterações no sistema real. A partir de um modelo, que descreve o comportamento do sistema a ser analisado, é possível realizar simulações e analisar seus resultados. A simulação tem um custo reduzido se comparado à mudanças reais no sistema (PRADO, 2010, FREITAS FILHO, 2008, LAW; KELTON, 2000).

Nesse contexto, este trabalho teve como objetivo analisar, por meio de simulação computacional, o processo de atendimento de um restaurante universitário. Optou-se por utilizar, neste trabalho, o software Arena ${ }^{\circledR}$ da Rockwell Software Corporation por ser um dos softwares de simulação discreta mais utilizado no mundo empresarial e acadêmico (PRADO, 2010).

O artigo é organizado da seguinte maneira. Na Seção 2 é descrita a metodologia empregada na simulação computacional. Na Seção 3 são apresentados os resultados obtidos da simulação do modelo do restaurante universitário. Comentários finais e conclusões, na Seção 4, finalizam o artigo.

\section{METODOLOGIA}

Tipo de Pesquisa:

A metodologia utilizada neste estudo pode ser classificada quanto ao tipo de pesquisa, a população amostra, a coleta e a análise dos dados. A classificação quanto ao tipo de pesquisa pode ser subdividida de acordo com a natureza sendo esta aplicada, quanto aos objetivos sendo descritiva, quanto à forma de abordar o problema pode ser considerada quantitativa e de acordo com os procedimentos técnicos como modelagem e simulação ou seja uma pesquisa operacional (YIN, 2001, GIL, 2002). 
Caracterização da Empresa:

O restaurante, objeto do estudo, está localizado na região oeste paranaense. O estabelecimento oferece diariamente almoço e janta para os alunos, servidores e moradores da região. As refeições são servidas pelo sistema self-service, compreendendo um cardápio com saladas e pratos quentes (composto por arroz, feijão, duas opções de carne e mistura), suco e sobremesa. No meses de março, abril e maio de 2016, por exemplo, foram servidas no restaurante 11170 refeições.

Processo de Atendimento:

$\mathrm{O}$ processo de atendimento inicia-se no momento que os clientes chegam ao restaurante universitário. Na sequência dirigem-se aos caixas. A seguir, deslocam-se para as mesas de buffet. Depois de se servirem deslocam-se para as mesas do restaurante. Na Figura 1 apresenta-se o fluxograma do processo.

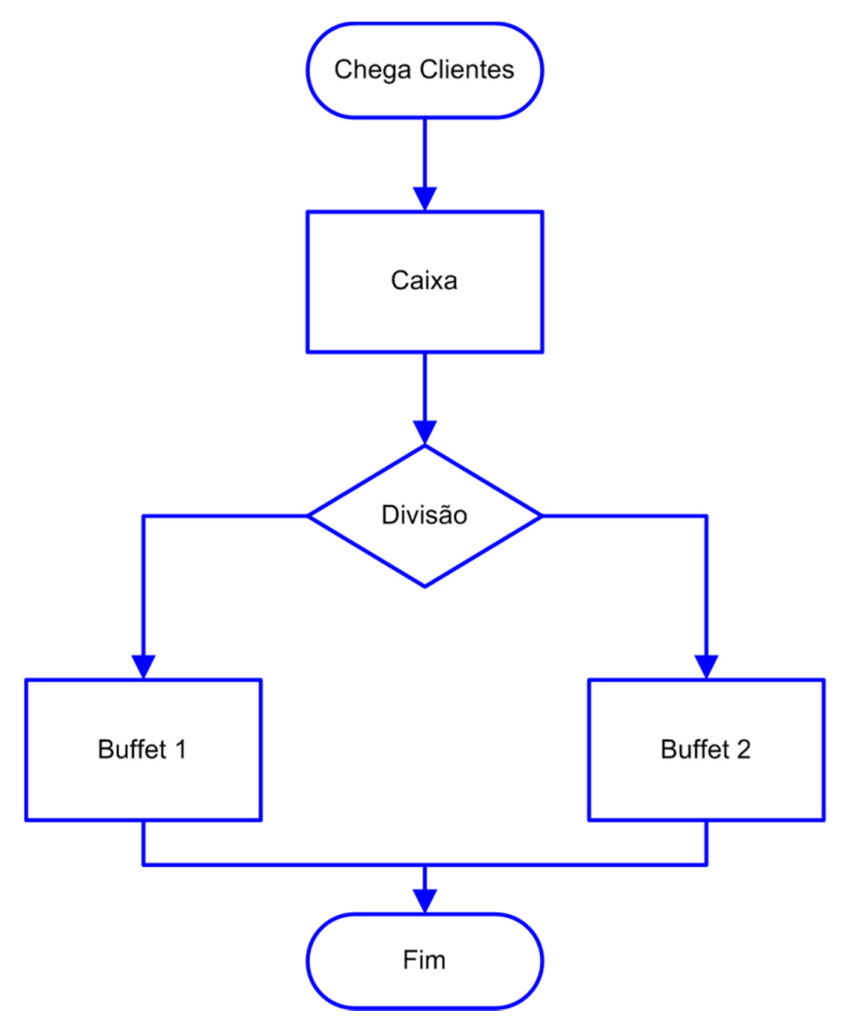

Figura 1: Fluxograma do processo de atendimento Fonte: $\mathrm{O}$ autor.

Coleta de Dados:

No planejamento, da coleta de dados, concluiu-se que seria necessário determinar as seguintes variáveis: Tempos entre chegadas dos clientes (TEC). Tempos de espera na fila do caixa (TF), Tempos de atendimento nos caixas (TC) e Tempos que os clientes levam nas mesas de buffet (TB). Utilizou-se a ferramenta Input Analyzer, do software Arena ${ }^{\circledR}$, para analisar alguns desses dados. O Input Analyzer permite analisar dados de funcionamento do sistema real e escolher a melhor distribuição teórica de probabilidades que se aplica a eles (PRADO, 2010; SOUTO JUNIOR, 2009).

Por meio do software Arena ${ }^{\circledR}$ student version realizou-se a simulação da dinâmica operacional do processo de atendimento. Nas suas ferramentas Output Analyzer e Process Analyzer analizaram-se os resultados de simulação. 
Número de Replicações: 1998):

Neste trabalho, o número de replicações ( $\mathrm{n}^{*}$ ) foi obtido através da Equação (1) (BANKS,

$$
\mathrm{n}^{*}=\mathrm{n} \times\left(\frac{\mathrm{h}}{\mathrm{h}^{*}}\right)^{2}
$$

onde:

n: número de replicações já realizadas;

h: semi-intervalo de confiança já obtido; e

$\mathrm{h}^{*}$ : semi-intervalo de confiança desejado.

Validação do Modelo:

$\mathrm{Na}$ execução do procedimento de validação, para o sistema em estudo, utilizou-se o erro médio estimado (Equação 2) (MONTGOMERY, 2005; SARGENT, 1998):

$$
\mathrm{SE}=\sqrt{\frac{(\mathrm{SR}-\mathrm{MD})^{2}}{\mathrm{GLR}}}
$$

onde:

SE - erro médio estimado;

$\mathrm{SR}$ - valor obtido a partir do sistema real;

MD - média dos valores gerados pelo modelo; e

GLR - grau de liberdade considerando o número de replicações do modelo.

Tamanho da Amostra:

O tamanho de cada uma das cinco amostras, cronometradas neste trabalho, foi obtida, para um nível de confiança de 95\%, através da Equação (3) (MARROCO, 2003):

$$
\mathrm{n}_{\mathrm{A}}=\left(\frac{\mathrm{Z \alpha /2} \times \mathrm{S}}{\mathrm{E}}\right)^{2}
$$

onde:

$\mathrm{n}_{\mathrm{A}}$ : número de indivíduos da amostra;

$\mathrm{Z}_{\alpha 2}$ : valor crítico que corresponde ao grau de confiança desejado;

S: desvio padrão;

E: erro máximo estimado. 
Produtividade do Processo:

Neste trabalho, a produtividade do processo de atendimento (PP), será medida por meio da Equação (4) (ZAHRAEE, 2016).

$$
\mathrm{PP}=\frac{\mathrm{Et}}{\mathrm{Sd}} \times 100
$$

Onde: $\mathrm{Et}$ - Número de clientes que entraram no restaurante e $\mathrm{Sd}-$ Número de clientes que sairam.

\section{RESULTADOS E DISCUSSÃO}

Inicialmentes, os dados obtidos no restaurante foram colocados na forma de boxplot (Figura 2). Na seqüência, a técnica de identificação de outliers foi aplicada (Tabela 1) (MARROCO, 2003). Estes valores surgem, geralmente, de erros na coleta de dados ou de eventos raros e inesperados. Observa-se, que os outliers só foram retirados das amostras somente depois de uma análise criteriosa. Mantiveram-se na amostra os valores possíveis de ocorrer.

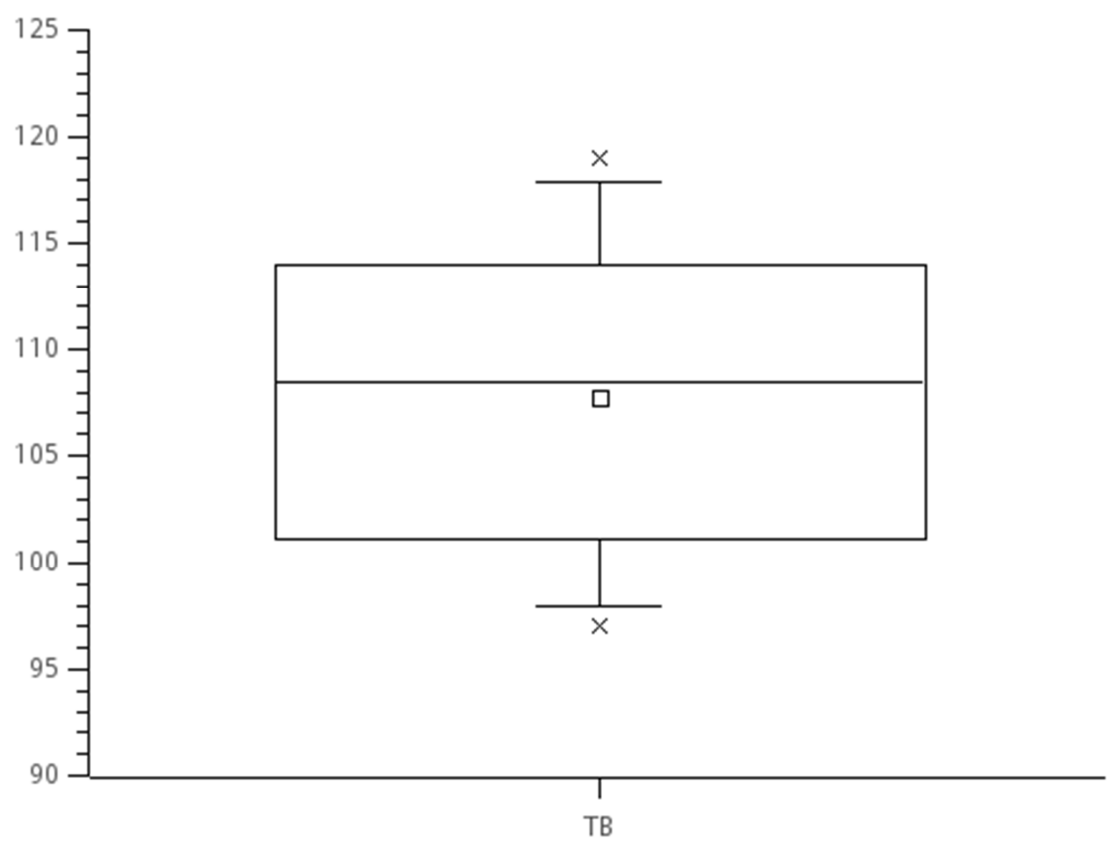

Figura 2: Boxplot dos tempos no buffet - TB

Fonte: $\mathrm{O}$ autor.

Tabela 1: Identificação de outliers

\begin{tabular}{l}
\hline \multicolumn{1}{c}{ Outliers } \\
\hline$A=Q^{3}-Q^{1}$ \\
Valor $<Q^{1}-1,5 A-$ Outlier Moderado \\
Valor $>Q^{3}+1,5 A$ - Outlier Moderado \\
Valor $<Q^{1}-3,0 A$ - Outlier Extremo \\
Valor $>Q^{3}+3,0 A$ - Outlier Extremo \\
Fonte: $O$ autor.
\end{tabular}


Onde

$\mathrm{A}=\mathrm{Q}^{3}-\mathrm{Q}^{1}$ : é a Amplitude inter-quartil;

$\mathrm{Q}^{1}$ : primeiro quartil;

$\mathrm{Q}^{3}$ : terceiro quartil.

A correlação entre os dados, ou seja a verificação da dependência entre os valores das amostras, foram analisadas. Na Figura 3, o gráfico de dispersão dos tempos no buffet é apresentado. Observa-se na Figura 3 que não existe correlação entre as observações da amostra.

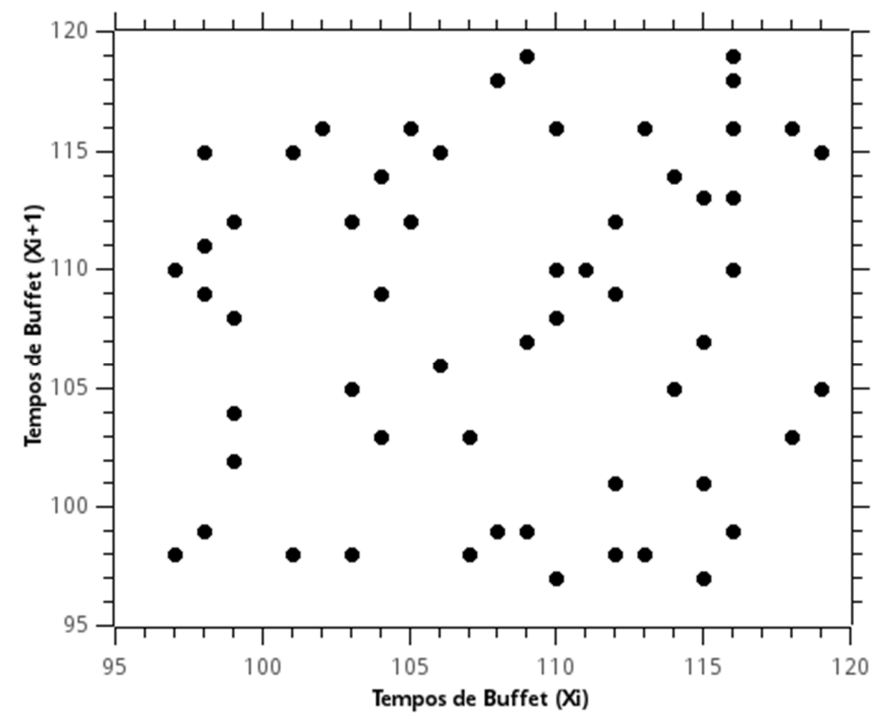

Figura 3: Gráfico de dispersão - tempos no buffet - TB

Fonte: $\mathrm{O}$ autor.

Na seqüência, as curvas de distribuições teórica de probabilidades foram determinadas por meio da ferramenta Input Analyzer do software Arena ${ }^{\circledR}$. Os $p$-values, dos testes de aderência Chi Square e Kolmogorov-Smirnof, apresentaram um nível de significância maior que o adotado $(0,1)$ (CHIWF; MEDINA, 2007; MARIN; TOMI, 2010). As distribuições que melhor aderiram aos dados coletados no sistema são apresentadas na Tabela 2.

Tabela 2: Distribuição de probabilidades

\begin{tabular}{cc}
\hline Iten & Distribuição \\
\hline TEC & $1.6999+\operatorname{EXPO}(7.95)$ \\
TC & $\operatorname{TRIA}(4,6.11,20.7)$ \\
TB & UNIF $(97,119)$ \\
\hline & Fonte: $O$ autor.
\end{tabular}

Fonte: $\mathrm{O}$ autor.

Na Figura 4 apresenta-se, como exemplo, o gráfico da distribuição de probabilidades dos tempos de buffet obtido por meio da ferramenta Input Analyser. 


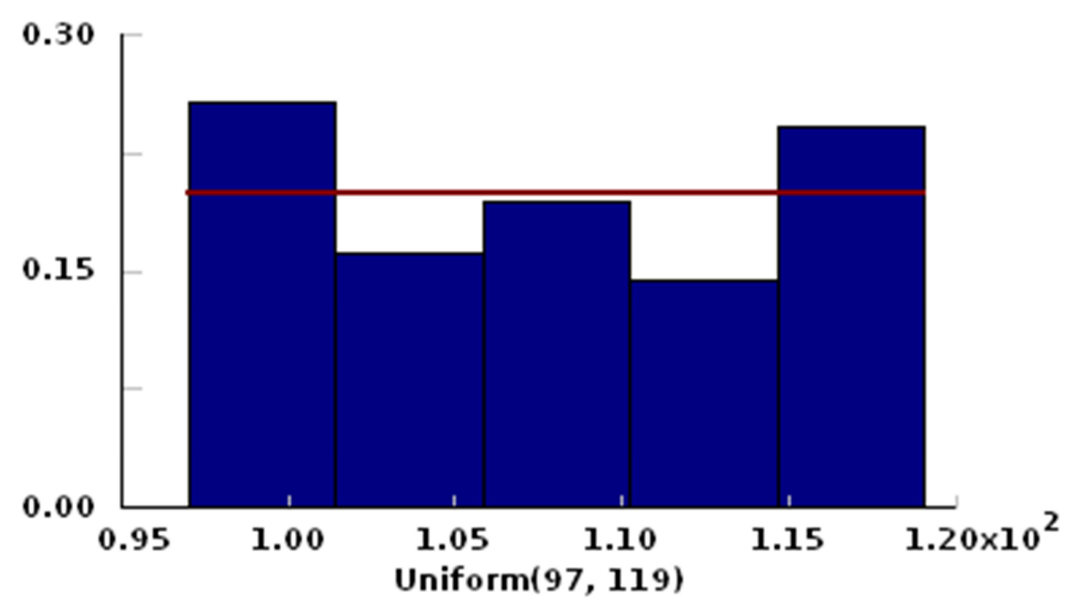

Figura 4: Distribuição de probabilidade - tempos no buffet - TB

Fonte: $\mathrm{O}$ autor.

Validação do Modelo Implementado:

Para validação do modelo do sistema em estudo realizou-se uma comparação (Tabela 3 ) entre o tempo médio de espera na fila do caixa, coletado no restaurante, com o tempo médio gerado pelo modelo de simulação. Na Tabela 3 apresenta-se, também, o erro médio estimado (SE, em decimal).

Tabela 3: Dados do sistema real e do modelo

\begin{tabular}{c|c|c}
\hline \multicolumn{3}{c}{ Tempo na Fila do Caixa (s) } \\
\hline Sistema Real & Modelo Computacional & SE \\
\hline 90,51 & $85,98 \pm 8,06$ & 0,82 \\
\hline \multicolumn{3}{c}{ Fonte: O autor. }
\end{tabular}

Na Figura 5 apresentam-se os boxplots dos dados do sistema real e do modelo computacional.

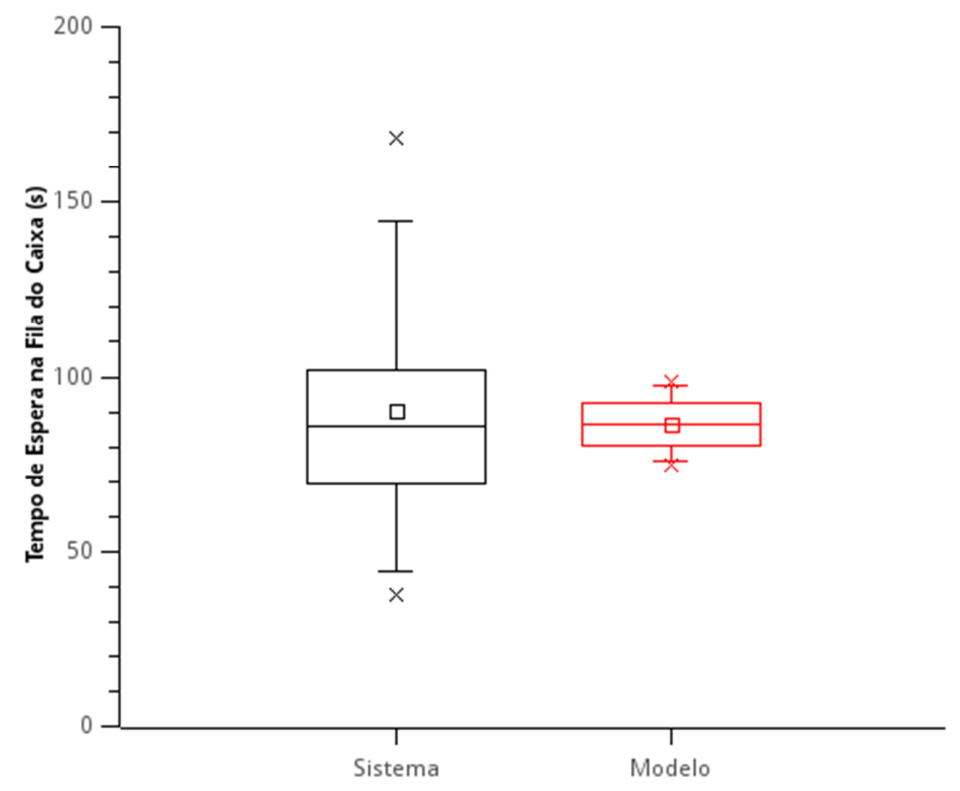

Figura 5: Boxplots dos dados do sistema real e do modelo Fonte: $\mathrm{O}$ autor. 
Na Figura 6 apresentam-se os resultados obtidos por meio do software Arena ${ }^{\circledR}$ paras as variáveis Número de Clientes na Fila do Caixa, Tempo na Fila do Caixa e Utilização do Caixa.

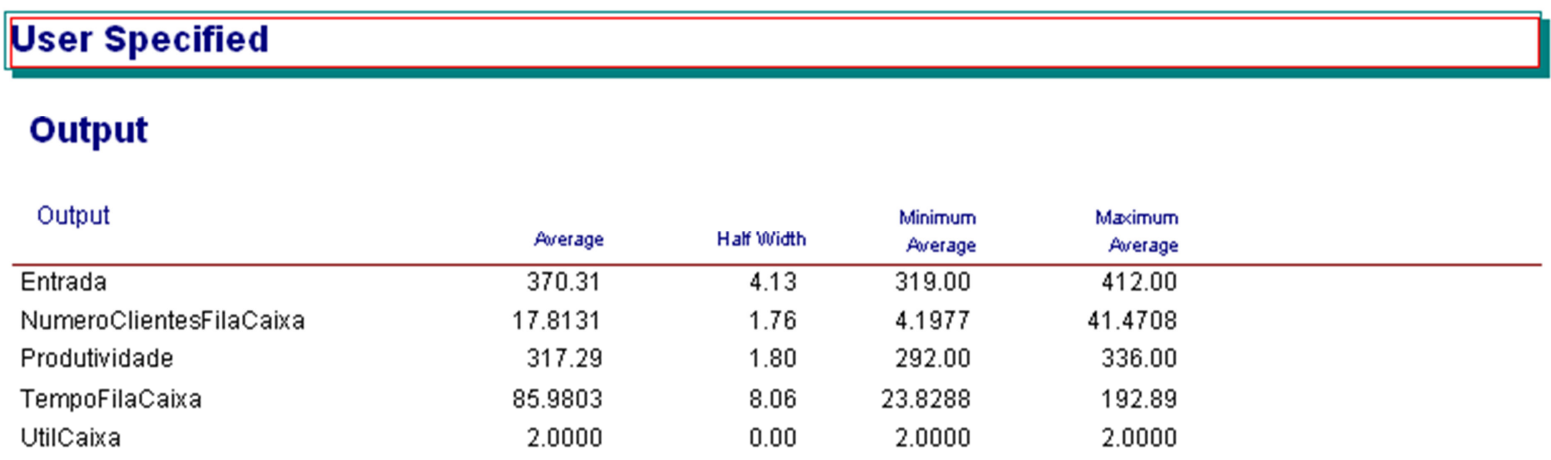

Figura 6: Resultados das variáveis obtidos do Software ARENA - 2 Caixas Fonte: $\mathrm{O}$ autor.

Simulação:

Para a realização da análise do sistema em estudo, são propostos dois cenários com o objetivo de observar a resposta do sistema a partir de alterações no número de caixas. O indicador de desempenho utilizado para a análise é a utilização dos caixas do restaurante.

- Cenário 1 (Cenário atual): 2 Caixas.

- Cenário 2: 3 Caixas.

A Tabela 5 apresenta os resultados obtidos da simulação do sistema, para os dois cenários.

Tabela 5: Resultados de simulação dos cenários 1 e 2

\begin{tabular}{c|c|c|c}
\hline Cenários & Caixa & Utilização & $\mathbf{N}^{\mathbf{0}}$ de Clientes na Fila (s) \\
\hline 1 & 2 & $100 \%$ & 18 \\
\hline 2 & 3 & $55,5 \%$ & 1 \\
\hline \multicolumn{4}{c}{ Fonte: O autor. }
\end{tabular}

Observa-se, dos resultados obtidos de simulação, que para o horário de pico (12:00 às 12:30 h) a utilização dos caixas chega a $100 \%$. Portanto, com a utilização de três caixas, neste período, reduziria a utilização dos caixas para $55,5 \%$ e o número de clientes na fila para 1 .

Em termos de produtividade, do processo de atendimento do restaurante, os resultados obtidos de simulação para os dois cenários foram os seguintes (Tabela 6):

Tabela 6: Resultados de produtividade do processo para os cenários 1 e 2

\begin{tabular}{c|c|c}
\hline Cenários & Caixa & Produtividade (PP) \\
\hline 1 & 2 & $85,68 \%$ \\
\hline 2 & 3 & $93,24 \%$ \\
\hline
\end{tabular}

Fonte: $\mathrm{O}$ autor. 
Percebe-e que, em termos de produtividade do processo, o aumento de 2 para 3 caixas não sofreu um ganho significativo $(7,56 \%)$, devido ao aumento de clientes nas filas das mesas do buffet. Portanto, o aumento de 2 para 3 caixas praticamente só teria efeito na utilização dos caixas, durante este pequeno período de tempo.

\section{CONCLUSÕES}

Neste trabalho apresentou-se a metodologia utilizada para implementar um modelo computacional utilizado para simular o processo de atendimento de um restaurante universitário localizado na região oeste paranaense.

De acordo com os resultados das análises procedidas para a validação do modelo computacional, foi possível concluir que o mesmo pode ser aplicado para simular a dinâmica operacional do processo de atendimento do restaurante universitário.

Observou-se, através dos resultados obtidos de simulação, que a ocupação dos caixas estava em torno de $100 \%$ durante o período simulado. Notou-se, também, que a inclusão de mais um caixa não surtiria gandes efeitos na produtividade do processo de atendimento. Sugere-se, aos responsáveis pelo restaurante, que seja deslocado um funcionário de outro setor para ajudar no atendimento dos caixas durante este pequeno intervalo de tempo.

Observa-se que estudos deste tipo, principalmente em pequenas e médias empresas tanto do setor industrial quanto de serviços, contribuem para a aproximação entre a universidade e estas empresas, promovendo o crescimento das empresas da região.

\section{REFERÊNCIAS}

ALVES, R.; SANTOS, J. A. A.; SCHMIDT, C. A. P. Aplicação dos princípios da teoria das restrições e de técnicas de simulação na gestão da dinâmica operacional de um pequeno restaurante: um estudo de caso. Revista Espacios, v. 35, p. 21, 2014.

BANKS, J. Discrete-event system simulation. New York. Prentice Hall, 2009.

CHWIF, L.; MEDINA, A. C. Modelagem e simulação de eventos discretos, teoria \& aplicações. São Paulo. Brazilian Books, 2007.

FREITAS FILHO, P. J. Introdução à Modelagem e Simulação de Sistemas com Aplicações em Arena. Florianópolis. Visual Books, 305 p., 2008.

GIL, A. C. Como elaborar Projetos de Pesquisa. 4. ed. São Paulo: Atlas, 2002.

LAW, A. M.; KELTON, W. D. Simulation modeling and analysis. New York. McGraw- Hill, 2000.

MARIN, T.; TOMI, G. F. C. Modelagem de dados de entrada para simulação estocástica del lavra. Revista Escola de Minas, v.60, 559-562, 2010.

MONTGOMERY, D. C. Design and Analysis of Experiments. New York. Wiley, 2005.

MARROCO, J. Análise estatística de dados - com utilização do SPSS. Lisboa. Sílabo, 2003.

PRADO, D. Usando o ARENA em simulação. Nova Lima. INDG - Tecnologia e Serviços LTDA, $307 \mathrm{p}, 2010$.

Persp. Online: exatas \& eng., Campos dos Goytacazes, 19 (07) 42-51 - 2017 
SARGENT, R. G. Verification and validation of simulation models. In: WINTER THE SIMULATION CONFERENCE, 1998, Washington. Proceedings... Washington: WSC, p. 20-28, 1998.

SOUTO JUNIOR, A. K. D. et al. Aplicação da simulação computacional para auxílio à tomada de decisão. In: XXIX Encontro Nacional de Engenharia de Produção, 2009, Anais... Salvador: ENEGEP, 2009.

YIN, R. K. Estudo de caso: planejamento e métodos. Porto Alegre: Bookman, 2001.

ZAHRAEE S. M., CHEGENI, A.; Toghtamish A. The Optimal Parameter Design for a Welding Unit of Manufacturing Industry by Taguchi Method and Computer Simulation. Journal of Industrial Engineering and Management, 2016. 